\title{
Two-photon microscopy to measure blood flow and concurrent brain cell activity
}

Running title: Blood flow in the brain

Corresponding author:

Dr. David Kleinfeld

Department of Physics

University of California 0374

9500 Gilman Drive

La Jolla, CA 92093-0374

E-mail: dk@physics.ucsd.edu 
Chapter 3.5 for "Optical Imaging of Cortical Dynamics", Bruno Weber and Fritjof Helmchen, eds.

\title{
Two-photon microscopy to measure blood flow and concurrent brain cell activity
}

\author{
Andy Y. Shih ${ }^{1}$, Jonathan D. Driscoll ${ }^{1}$, Michael J. Pesavento ${ }^{1}$ and David Kleinfeld ${ }^{1,2}$ \\ ${ }^{1}$ Department of Physics, Division of Physical Sciences, University of California at San Diego, \\ La Jolla, CA 92037 USA \\ ${ }^{2}$ Section on Neurobiology, Division of Biological Sciences, University of California at San \\ Diego, La Jolla, CA 92037 USA
}

\begin{abstract}
The cerebral vascular system services the constant demand for energy during neuronal activity in the brain. Attempts to delineate the logic of neurovascular coupling have been greatly aided by the advent of two-photon laser scanning microscopy to concurrently image blood flow and the activity of individual neurons and astrocytes involved in the control of the flow. Here we review the procedures to generate optical access to the cortex for both rats and mice, determine the receptive fields of the exposed areas, and use two-photon microscopy to accurately measure blood flow in individual cortical vessels concurrent with local cellular activity. We illustrate the techniques with acute recordings from rats and chronic recordings from mice.
\end{abstract}

Keywords: Astrocytes, flux, neurons, scanning, vasculature, vasomotion 


\section{Introduction}

Blood is a vital and limited resource in the brain. All aspects of neuronal and non-neuronal activity require a supply of oxygen and glucose - a need that constantly evolves with changes in brain activity $(1,2)$. How is the distribution of blood controlled relative to these changing needs? Delimiting this phenomenon, commonly termed functional hyperemia or neurovascular coupling, remains an active area of research (3). However, recent studies have highlighted important conditions under which neural activity and blood flow become decoupled (4-6), and thus raise basic questions about neurovascular coupling (7). One set of questions concerns the patterns of neuronal signals that lead to vasoactivity. A second set concerns the astrocytes that ensheath the vasculature and their role as intermediary cells that deliver signals from neurons to blood vessels.

The answer to the above questions depends on the ability to image blood flow and cells throughout the depth of cortex, 1.2 to $1.5 \mathrm{~mm}$ in rat and 1.0 to $1.2 \mathrm{~mm}$ in mouse. Imaging at this depth with subcellular resolution is facilitated by two-photon laser scanning microscopy (8), an optical sectioning technique in which absorption of light to excite fluorescent molecules occurs only at the laser focus. Past studies have made use of two-photon microscopy to examine vascular dynamics and blood flow in multiple brain regions, including somatosensory cortex (4, 9-27) and the olfactory bulb $(6,28-31)$, down to depths of $600 \mu \mathrm{m}$, which is sufficient to resolve vessels and neurons in layer 4. Recent advancements show that two-photon microscopy can achieve imaging depths that allow single microvessels to be studied throughout the full depth of cortex (32), and neuronal dynamics down to layer 5b (33), which is an important issue since vascular regulation in cortex appears to initiate in middle and deeper layers of cortex (9). A further advantage is the concomitant use of exogenous and endogenous fluorescence-based functional reporters to observe cellular activity, such as changes in intracellular $\mathrm{Ca}^{2+}$ 
concentration $(22,28,31)$, and the ratio of NADP to $\operatorname{NAD}^{+}(34,35)$ concurrent with blood flow and vessel diameter changes.

Here we discuss basic procedures of single and multi-vessel two-photon imaging of blood flow dynamics, concurrent with cellular activity, in the somatosensory cortex of anesthetized and awake rodents. We further provide case studies. The equipment and algorithms used in these studies have been summarized elsewhere, including comprehensive reviews of basic methodology of vascular imaging $(36)$, hardware $(37,38)$ and software $(39,40)$ for two-photon microscopy, and algorithms for data analysis (41-44). Additional work has addressed the use of two-photon microscopy to image histological tissue with labeled vasculature $(7,45-48)$.

\subsection{Brain window preparation}

Both rats and mice have their place in cerebral blood flow imaging studies. The relatively large size of rats allows them to tolerate anesthesia better than mice and makes them the animal of choice for complex surgical procedures. Extended cranial windows can be fabricated to permit access to multiple regions of cortex, blood samples may be obtained at multiple time points in a procedure, and physiological parameters can be readily controlled. However, a current disadvantage of cranial windows, and thus rats, is that the imaging quality degrades within days. The use of mice has two advantages. First, they allow researchers to exploit the wide range of vascular related transgenic animals. Second, transcranial windows with a thinned skull may be fabricated. The use of transcranial windows obviates potential problems with inflammation and changes in cranial volume and is excellent for repeated imaging studies over many months. On the down side, blood sampling and physiological control is limited with mice compared with rats. 
Cranial windows. The generation of a cranial window for optical access in rats and mice differs on a number of levels. In rats, the overlying bone must be completely removed. Further, the dura mater must be carefully resected to the edge of the imaging window for optical access (49-51). The window must then be resealed to restore intracranial pressure and to minimize motion artifacts caused by heart beat and breathing. Very large windows can be generated, i.e., 4 × $6 \mathrm{~mm}$, to facilitate easy positioning of electrodes and cannulas. The clarity through such a cranial window is initially optimal as the materials overlying the pial surface cause minimal scattering. However, dural regrowth degrades the imaging quality such that repeated imaging is limited, in our hands, to about four days. As a result, chronically implanted windows for repeated imaging in rats are rarely reported and the use of pharmacological agents to suppress inflammation may also affect the phenomenon under study, such as the magnitude of injury in experimental stroke models (52). Longer lasting windows may, in principle, be achieved by using inert substances such as Kwiksil silicone for movement suppression (53).

Cranial windows in mice are surgically less demanding, as the dura is thin and does not need to be removed for optical access. Detailed methods have been described for cranial windows $(54,55)$ and transcranial, thinned skull windows $(56)$.

Transcranial windows. An alternate method that is suitable for mice is to generate a stable transcranial window, where the skull is thinned, polished and reinforced with a thin layer of glue and cover slip $(57,58)$. These windows, which may be as large as $2 \times 2 \mathrm{~mm}$, greatly minimize disruption of the intracranial milieu, reduce inflammation, and prevent bone regrowth. While the imaging depth and clarity are somewhat reduced compared to windows with complete bone removal, polished and reinforced cranial windows in mice have been proven to give excellent clarity for two-photon imaging months after the initial surgery. This procedure has so far failed 
with rats because of the lower clarity of the dura and skull.

\subsection{Localization of active areas}

Standard brain atlases provide approximate coordinates for different brain regions as a means to locate the vasculature in relation to loci of neuronal activity. Yet somatotopically refined maps are often of considerable value. The lissencephalic structure of the rodent brain permits different regions in cortex to be further mapped to determine receptive fields using a variety of classical tools. For sensory areas, these include surface electrodes and intrinsic optical signal (IOS) imaging. The latter technique avoids any contact with the brain and uses changes in the intensity of reflected light to report a change in the ratio of oxy- to deoxyhemoglobin that occurs secondary to changes in neuronal activity $(59,60)$. Motor areas may also be mapped in one of three ways: (i) measuring limb or vibrissa movement in response to intracortical microstimulation with bipolar microelectrodes; (ii) measuring electromyogenic activity in response to intracortical microstimulation; or (iii) through the use of focal illumination in conjunction with mice that express channelrhodopsin in projection neurons (61).

We illustrate the mapping process with IOS imaging for the case of vibrissa primary sensory cortex (Fig. 1). A craniotomy was prepared, as described previously (49), and individual vibrissae on the face of the animals were mechanically stimulated. Stimulation of a single vibrissa leads to a net decrease in reflectance at $633 \mathrm{~nm}$ within a localized region of vibrissa primary sensory cortex (Fig. 1A). This corresponds to a local decrease in blood oxygenation. The reflectance, denoted $R_{i}(x, y, t)$ where $i$ labels the trial, was quantified as a function of time and trial. We computed the smoothed, trial-averaged value as $\mathrm{R}(\mathrm{x}, \mathrm{y}, \mathrm{t}) \equiv \operatorname{Gaus\operatorname {sian}}\left[<\mathrm{R}_{\mathrm{i}}(\mathrm{x}, \mathrm{y}, \mathrm{t})\right.$ $\left.\operatorname{median}[\mathrm{R}(\mathrm{x}, \mathrm{y}, \mathrm{t})]>_{\text {trial }}\right]$ where the Gaussian filter had a width of $\sigma=4$ pixels. For the data of

figure 1 , the duty cycle for each trial was $18 \mathrm{~s}$, and we obtained 30 trials. The initial $4 \mathrm{~s}$ of the 
average signal constitutes baseline, i.e., $\mathrm{R}_{\text {base }}(\mathrm{x}, \mathrm{y}) \equiv<\mathrm{R}(\mathrm{x}, \mathrm{y}, \mathrm{t})>_{0 \mathrm{~s} \leq \mathrm{t} \leq \mathrm{s}}$, the second $4 \mathrm{~s}$ were used to deflect individual vibrissae, and a period after the onset of stimulation was used to form $\mathrm{R}_{\text {response }}(\mathrm{x}, \mathrm{y}) \equiv<\mathrm{R}(\mathrm{x}, \mathrm{y}, \mathrm{t})>_{4.5 s \leq \leq \leq \leq 9 s}$. Finally, the $\operatorname{IOS}$ is expressed as $\Delta \mathrm{R} / \mathrm{R} \equiv\left[\mathrm{R}_{\text {response }}(\mathrm{x}, \mathrm{y})-\right.$ $\left.R_{\text {base }}(x, y)\right] / R_{\text {base }}(x, y)$. The centroid of this signal and the overall shape of the active region remain roughly constant between blocks of trials (Fig. 1B), although the amplitude varies greatly between blocks. Nonetheless, IOS imaging serves as a useful tool for mapping the center of activation in cortex for the different vibrissa (Fig. 1C). The branching arrangement of pial arterioles and venules, which is unique to each animal, serves as fiducials to relocate the active region between intrinsic imaging and two-photon measurements.

\section{Blood flow measurements}

\subsection{Measurement of blood flow dynamics in single cortical vessels}

We consider first the measurement of the flux of blood flow. This involves a user-defined scan profile for two-photon microscopy to simultaneously measure both the speed of red blood cells (RBCs) and the diameter of vessels as a means to compute the volume flux of RBCs. The vasculature is labeled with an intravenous bolus of fluorescein- or rhodamine-conjugated dextran for green and red light emission, respectively. The cerebral vasculature within the window is first imaged at low resolution with a 5-times magnification objective (Fig. 2A); this image can be directly compared with that made with IOS imaging (Fig. 1C). High resolution imaging of surface pial vessels, penetrating vessels, and subsurface capillaries can then be performed in smaller regions with a high numerical aperture water dipping objective, such as a 20-times or 40times magnification objective with numerical apertures ranging from 0.7 to 1.0 (Fig. 2B). 
When the serum is labeled, RBCs exclude the dextran dye and appear as dark objects moving against a bright fluorescent background. We used custom software to direct the imaging laser beam in a user-defined path within the imaging plane (Box 1) $(42,44)$. Linear segments of constant scan speed traverse along the length of the center of the vessel and across the width of the vessel to measure RBC speed and lumen diameter, respectively. These linear scan segments are connected by polynomial splines, where connecting portions of the scan are accelerated to allow for rapid data collection across multiple vessels (44) (Fig. 2C).

The resulting line scan data forms a space-time image, typically displayed with the individual scan lines stacked on each other (Fig. 2D). In principle, many vessels that lie in the same plane can be measured simultaneously. Portions of the scan path along the centerline of the vessel lumen reveal angled streaks within the cascade image. Moving RBCs in flowing vessels sampled at a sufficient rate will appear as diagonal streaks. The centerline velocity is proportional to the slope of the RBC streaks, measured from vertical (Fig. 2E). Determining this slope is most efficiently and robustly performed with a Radon transform of the data (41).

A velocity time series is calculated by transforming successive time-windowed portions of the line scans (right panel in Fig. 2F). The temporal spacing of successive windows must be close enough to resolve the highest velocity modulation frequency, the heart rate, which is $\sim 6 \mathrm{~Hz}$ for rats and $\sim 10 \mathrm{~Hz}$ for mice. In addition, the window size must be large enough to capture enough streak lines so that the Radon transform has sufficient data to calculate an accurate velocity value. We find that a window size of $40 \mathrm{~ms}$ with a spacing of $10 \mathrm{~ms}$ is a good compromise. In addition to heart rate, other physiological signals detected in the RBC velocity time series include breathing, at $\sim 1 \mathrm{~Hz}$ for rats and $\sim 2 \mathrm{~Hz}$ for mice, and vasomotion at $\sim 0.1 \mathrm{~Hz}$ for rat and 0.1 to $1 \mathrm{~Hz}$ for mice $(16,43,62)$. 
As with the velocity calculation, the diameter calculation is taken from a time-windowed portion of the data (Fig. 2D). The same window size and spacing used for velocity is also used for diameter so that both parameters are concurrently calculated. Vessel diameter is defined as full width at half maximum of the vessel profile for each window (left panel in Fig. 2E); the intensity profile tends to increase near the edges due to the exclusion of RBCs from the glycocalyx and endothelial surface layer. The two outermost half maximal points of these peaks are used to calculate the vessel boundary. Linear interpolation is used to add subpixel accuracy to the diameter measurement.

When the diameter of the vessel is much greater than that of the $\mathrm{RBC}$, the flow is laminar and nearly parabolic $(14,63)$. The two vascular parameters, RBC velocity and lumen diameter, are combined to calculate the volume flux, RBCs and plasma, for each vessel. The volume flux through the vessel is given by

$$
\overrightarrow{\mathrm{F}}=\langle\overrightarrow{\mathrm{v}}\rangle A=\frac{\pi}{8} \overrightarrow{\mathrm{v}}(0) \mathrm{d}^{2}
$$

where $\vec{v}(0)$ is the time averaged RBC velocity at the center line of the vessel, $A$ is the cross sectional area of the vessel lumen, and $d$ is the lumen diameter. This formula underestimates the flux as the nonzero spatial extent of the RBC flattens the parabola of Poiseuille flow.

As an example, we measure the change in flux at the level of single penetrating vessels in response to somatosensory stimulation. These vessels deliver blood from the communicating vessels on the surface of cortex to the subsurface microvessls (15). Both diameter and RBC velocity in the arteriole respond to stimulation (left column in Fig. 2F). The flux through the arteriole increases to a peak of $86 \%$ over baseline, compared with a much smaller peak increase of $29 \%$ and $24 \%$ for diameter and velocity measurements alone, respectively. The increase in 
$\mathrm{RBC}$ velocity is partially masked by a peak in the underlying vasomotor fluctuation, but remains a significant increase over an average one minute period of basal activity. In contrast to the arteriole, a neighboring venule exhibits no change in lumen diameter, but a $23 \%$ change in RBC velocity. As a result, the flux increases in the venule by $23 \%$ as well (right column in Fig. 2F). This increase in venous flux would not be detected with methods that measure diameter only.

\subsection{Simultaneous imaging of blood flow and local cellular activity}

An important goal is to identify the cell types and signaling pathways that regulate neurovascular coupling. The activity of various neuronal cell types and astrocytes can be monitored in superficial cortex by bulk loading the tissue with functional dyes, in this case Oregon Green Bapta-1 AM (OGB-1 AM), to detect changes in intracellular $\mathrm{Ca}^{2+}$ concentration $(64,65)$ (Fig. 3A) (see chapter $\mathrm{xx}$ in this volume). The $\mathrm{Ca}^{2+}$ indicators may be injected by pipette into the active region through a vent in the cranial window under two-photon guidance, or prior to sealing the window during the initial craniotomy procedure. Astrocytes are selectively labeled with the astrocytic marker sulforhodamine 101 (SR101) in the same tissue to distinguish them from neurons in a second imaging channel (66).

We consider a particular field of view that contains 20 identified cells, i.e., 19 neurons and one astrocyte, along with three blood vessels. Even at this level it is tedious to localize each labeled cell and guide the laser scan path manually. We thus used a machine learning algorithm to locate each soma (42) (Box 1) in conjunction with full-field images from the OGB-1 emission channel. Cells that were colabeled with SR101 were automatically labeled as astrocytes; the coordinates of selected blood vessels were also marked. We then calculated the fastest scan path through each soma along with selected microvessels (Fig. 3A) (Box 1). The typical signal-toRMS-noise ratio, which we define as the ratio of the peak of the response to the RMS noise 
during the baseline, for a change in intracellular $\mathrm{Ca}^{2+}$ induced by a single sensory stimulus is $\sim 10$ (Fig. 3B). Further, the resulting line scan image contains stereotypical angled streaks for calculation of RBC speed, as well as intensity traces from selected neurons and astrocytes, similar to that seen in Figure 2C. The composite data permits comparison of changes in astrocytic $\mathrm{Ca}^{2+}$ levels, together with changes in the speed of RBCs in a nearby microvessel, with the composite neuronal activity (Fig. 3C).

\subsection{Stimulated and basal hemodynamics in awake mice}

We consider the quantification of blood flow both at and below the cortical surface in awake mice. Mice were habituated to head fixation and blood flow was measured in single vessels through a chronically implanted transcranial window (Fig. 4A). Robust arteriole dilations could be evoked by prolonged contralateral whisker stimulation (Figs. 4B and 4C, red). Small arterioles dilated proportionally more than larger arterioles and the rapid dilation that followed vibrissa simulation and vibrissae evoked dilation (Figs. 4Aand 4B). In contrast, the delayed, second peak in the dilation does not show a significant dependence on vessel size (Fig. 4B), nor does the response to control stimuli (Fig. 4C). Pial venules, typically thought to be static in terms of diameter, show a delayed and weak dilation in the awake state that had no dependence on initial diameter (Fig. 4C). These data suggest that functional hyperemia changes detected by BOLD fMRI contain a significant and possibly dominant contribution from large changes in arteriole volume, in concurrence with recent studies (67), rather than in venules (68).

Individual traces of the arteriole diameter show a high degree of variation and spontaneous dilation (Fig. 4B). Similar to the case of stimulus-induced changes, small arterioles dilated proportionally more than larger arterioles for both spontaneous events (Fig. 4D). The 
critical point raised by this data is that the magnitude of both prompt and delayed arterial responses induced by stimulation are similar in magnitude to spontaneous arterial dilations, with typical dilations of $30 \%$ and maximum dilations near $50 \%$ for both cases (Figs. 4B to 4D). Thus, from the point of control, stimulus-induced changes in blood flow are small, i.e., on the order of the noise level. The corollary is that individual stimulus events cannot be distinguished from spontaneous dilations based on magnitude alone $(20,69)$.

\subsection{Imaging of blood flow in deep cortical layers}

An important development in two-photon microscopy is the ability to image deep cortical layers that are responsible for the output of neuronal processing (70). Further, different cell types predominate in different layers, so that deep imaging permits neurovascular coupling to be studied in changing environments. Longer wavelengths of light penetrate deeper into tissue as a result of reduced scattering, absorption, and optical aberration by the tissue. In the example of

Figure 5, a cranial window was prepared in a mouse and the dye Alexa 680-conjugated dextran was used to label the blood serum. The dye was excited with $1280 \mathrm{~nm}$ wavelength light from a Ti:sapphire pumped optical parametric oscillator and was found to enable imaging through the entire depth of cortex, i.e., $1 \mathrm{~mm}$ deep (Figs. 5A and 5B) (32). Red blood cell velocities in capillaries could be resolved as deep as $900 \mu \mathrm{m}$ into cortex (Figs. 5C and 5D).

\section{Summary}

Two-photon microscopy provides a number of advantages that will aid the study of the mechanisms underlying neurovascular coupling and cerebrovascular disease in animal models, 
including $i$ ) the resolution needed to visualize single cortical vessels and their surrounding cells, ii) penetration depths of $250 \mu \mathrm{m}$ through a transcranial window and $500 \mu \mathrm{m}$ with craniotomies at $800 \mathrm{~nm}$ excitation, and imaging to $1000 \mu \mathrm{m}$ depth with longer excitation wavelengths, iii) reduced photodamage, $i v$ ) high speed user-defined line scans for near simultaneous measurement of $\mathrm{RBC}$ velocity, lumen diameter and local cellular activity, and v) the opportunity to image vascular dynamics deep in the cortex of awake mice. Advances in two-photon microscopy that enable rapid line scans in all three dimensions (71), as opposed to just within a plane, will enable real-time estimates of global flow within a region.

A wide variety of functional fluorescent dyes can be exploited for studies of neurovascular control. Recent focus has been on $\mathrm{Ca}^{2+}$ sensors to study cellular activity. Exogenous sensors have the advantage of labeling both neurons and astrocytes, along with a meshwork of processes that intervene between cell bodies. However, the cellular source of a signal may be difficult to resolve when focusing on fine processes. Transgenic mice with different neuronal subtypes labeled with fluorescent proteins can aid the separation of responses from different cell populations (72). Genetically encoded $\mathrm{Ca}^{2+}$ sensors exclusively expressed in a specific cell type can be essential in separating signals from individual cells (73). Finally, cyclic adenosine monophosphate sensors would also be of value since $\mathrm{Ca}^{2+}$ independent pathways may also be involved in neurovascular control.

The intermixing of cell types within a small tissue volume also hinders electrophysiological and pharmacological approaches to query the role of cell types in neurovascular coupling. New advances with light-activated opsins (74) or engineered receptors with unnatural affinities for exogenous chemicals (75) may help to unravel issues with cellular specificity (7). However, caveats should also be considered, as activation of one cell type does 
not preclude activation of non-targeted cells linked within the same circuitry. The manipulation of cell-specific vasoactive signaling cascades will be an important step in dissecting the chemical basis of neurovascular coupling, but will also be challenging as new tools to knockdown gene expression will need to be developed (7). 


\section{Box 1 Arbitrary scan patterns through automatically selected targets}

In order to generate a standard two-dimensional two-photon microscopy image, the laser focal point is scanned systematically across the entire field of view in a raster pattern. Repeating the scan pattern generates a time series of frames and results in an easy to interpret movie of the entire field of view or a zoom of a smaller region. However, in many cases the relevant optical signal is spread through small portions of the field of view, such as when a sparse network of labeled neurons fire in response to stimuli. In such cases, it is advantageous to scan primarily across the active regions of interest, to maximize the number of signal photons acquired per unit of time. This not only maximizes the signal to noise ratio, but allows imaging on much faster timescales than the typical raster scan frame rates.

Frequently, the field of view contains only a few obvious neurons, or regions of interest (ROIs),

which can be selected by the user during the experiment. In cases where a large number of ROIs are present, such as a sparse network of labeled neurons which are responsive to stimuli, machine learning can be used to automatically detect the regions and create an optimized scan path which passes through all of them in a minimum amount of time (Fig. 6). Typically, a computer algorithm is trained on experimental data which has been annotated by a human to mark relevant areas that the algorithm learns to recognize. During an experiment, the algorithm can then be used to quickly and automatically identify the ROIs. A typical workflow is (42):

- A region of cortex is labeled with a $\mathrm{Ca}^{2+}$ indicator. An initial, full frame movie is collected while the animal is stimulated so that a subset of the cells responds with increased fluorescence. Multiple training and validation datasets can be created by taking multiple movies over the same or similar regions.

- After the experiment, the movies are marked by a human expert. Regions are labeled as a "cell" or "possibly a cell". Unmarked regions are considered to be "not a cell." This training data set is used to create a pixel classifier, which is used to determine if a given pixel is or is not a cell, and a "blob" classifier, which uses the shapes of pixels to determine actual cell regions. The data is used 
to generate a set of mathematical rules, which can be implemented by the computer, to form a basis for the classifiers (76). The classifiers operate on eight features in the dataset: the mean, variance, covariance, and correlation contained in the images, as well as the mean, variance, covariance, and correlation normalized by the standard deviation. After identifying pixels which are likely to belong to a cell, a morphological classifier using features such as size and eccentricity are used to delineate intact cells.

- During subsequent experiments, a movie is taken of a region similar to that used in the original experiment. This movie, along with the classifiers created previously, is fed into the software, which automatically generates a set of ROIs along, with an initial laser scan path across them. The ROIs can then be modified by the user, typically by adding or removing ROIs.

- The time it takes to scan across all the ROIs will depend on the order in which they are scanned. Optimizing the order is the classic "traveling salesman" problem, which has been extensively studied in computer science and mathematics. In general, it is not possible to search through all possible paths, and some computational shortcuts are taken. We use the ANT optimization algorithm to search rapidly through a subset of all possible paths as a means to find the shortest path that travels through all regions (77). 


\section{References}

1. Fox, P. T., and Raichle, M. E. (1986) Focal physiological uncoupling of cerebral blood flow and oxidative metabolism during domatosensory stimulation in human subjects, Proceedings of the National Academy of Sciences USA 83, 1140-1144.

2. Leybaert, L. (2005) Neurobarrier coupling in the brain: A partner of neurovascular and neurometabolic coupling?, Journal of Cerebral Blood Flow \& Metabolism 25, 2-16.

3. Attwell, D., Buchan, A. M., Charpak, S., Lauritzen, M., MacVicar, B. A., and Newman, E. A. (2010) Glial and neuronal control of brain blood flow, Nature 468, 232-243.

4. Devor, A., Hillman, E. M., Tian, P., Waeber, C., Teng, I. C., Ruvinskaya, L., Shalinsky, M. H., Zhu, H., Haslinger, R. H., Narayanan, S. N., Ulbert, I., Dunn, A. K., Lo, E. H., Rosen, B. R., Dale, A. M., Kleinfeld, D., and Boas, D. A. (2008) Stimulus-induced changes in blood flow and 2deoxyglucose uptake dissociate in ipsilateral somatosensory cortex, Journal of Neuroscience 28, 14347-14357.

5. Sirotin, Y. B., and Das, A. (2008) Anticipatory haemodynamic signals in sensory cortex not predicted by local neuronal activity, Nature 457, 475-479.

6. Jukovskaya, N., Tiret, P., Lecoq, J., and Charpak, S. (2011) What does local functional hyperemia tell about local neuronal activation?, Journal of Neuroscience 31, 1579-1582.

7. Kleinfeld, D., Blinder, P., Drew, P. J., Driscoll, J. D., Muller, A., Tsai, P. S., and Shih, A. Y. (2011) A guide to delineate the logic of neurovascular signaling in the brain, Frontiers in Neuroenergetics 3, 1-9.

8. Denk, W., Strickler, J. H., and Webb, W. W. (1990) Two-photon laser scanning fluorescence microscopy, Science 248, 73-76.

9. Tian, P., Teng, I., May, L. D., Kurz, R., Lu, K., Scadeng, M., Hillman, E. M., De Crespigny, A. J., D'Arceuil, H. E., Mandeville, J. B., Marota, J. J., Rosen, B. R., Lui, T. T., Boas, D. A., Buxton, R. B., Dale, A. M., and Devor, A. (2010) Cortical depth-specific microvascular dilation underlies laminar differences in blood oxygenation level-dependendent functional MRI signal, Proceedings of the National Academy of Sciences USA 107, 15246-15251.

10. Sigler, A., Mohajerani, M. H., and Murphy, T. H. (2009) Imaging rapid redistribution of sensoryevoked depolarization through existing cortical pathways after targeted stroke in mice, Proceedings of the National Academy of Sciences USA 106, 11758-11764.

11. Zhang, S., and Murphy, T. H. (2007) Imaging the impact of cortical microcirculation on synaptic structure and sensory-evoked hemodynamic responses in vivo, Public Library of Science Biology 5, e119.

12. Brown, C. E., Li, P., Boyd, J. D., Delaney, K. R., and Murphy, T. H. (2007) Extensive turnover of dendritic spines and vascular remodeling in cortical tissues recovering from stroke, Journal of Neuroscience 27, 4101-4109.

13. Zhang, S., Boyd, J., Delaney, K. R., and Murphy, T. H. (2005) Rapid reversible changes in dendritic spine structure in vivo gated by the degree of ischemia, Journal of Neuroscience 25, 5333-5228.

14. Schaffer, C. B., Friedman, B., Nishimura, N., Schroeder, L. F., Tsai, P. S., Ebner, F. F., Lyden, P. D., and Kleinfeld, D. (2006) Two-photon imaging of cortical surface microvessels reveals a robust redistribution in blood flow after vascular occlusion, Public Library of Science Biology 4, 258270.

15. Nishimura, N., Schaffer, C. B., Friedman, B., Lyden, P. D., and Kleinfeld, D. (2007) Penetrating arterioles are a bottleneck in the perfusion of neocortex, Proceedings of the National Academy of Sciences USA 104, 365-370.

16. Drew, P. J., Shih, A. Y., and Kleinfeld, D. (2011) Fluctuating and sensory-induced vasodynamics in rodent cortex extends arteriole capacity, Proceedings of the National Academy of Sciences USA $108,8473-8478$. 
17. Blinder, P., Shih, A. Y., Rafie, C. A., and Kleinfeld, D. (2010) Topological basis for the robust distribution of blood to rodent neocortex, Proceedings of the National Academy of Sciences USA 107, 12670-12675.

18. Shih, A. Y., Friedman, B., Drew, P. J., Tsai, P. S., Lyden, P. D., and Kleinfeld, D. (2009) Active dilation of penetrating arterioles restores red blood cell flux to penumbral neocortex after focal stroke, Journal of Cerebral Blood Flow \& Metabolism 29, 738-751.

19. Winship, I. R., Plaa, N., and Murphy, T. H. (2007) Rapid astrocyte calcium signals correlate with neuronal activity and onset of the hemodynamic response in vivo, Journal of Neuroscience 27, 6268-6272.

20. Kleinfeld, D., Mitra, P. P., Helmchen, F., and Denk, W. (1998) Fluctuations and stimulus-induced changes in blood flow observed in individual capillaries in layers 2 through 4 of rat neocortex, Proceedings of the National Academy of Sciences USA 95, 15741-15746.

21. Devor, A., Tian, P., Nishimura, N., Teng, I. C., Hillman, E. M., Narayanan, S. N., Ulbert, I., Boas, D. A., Kleinfeld, D., and Dale, A. M. (2007) Suppressed neuronal activity and concurrent arteriolar vasoconstriction may explain negative blood oxygenation level-dependent signaling, Journal of Neuroscience 27, 4452-4459.

22. Wang, X., Lou, N., Xu, Q., Tian, G. F., Peng, W. G., Han, X., Kang, J., Takano, T., and Nedergaard, M. (2006) Astrocytic $\mathrm{Ca}^{2+}$ signaling evoked by sensory stimulation in vivo, Nature Neuroscience 9, 816-823.

23. Fernández-Klett, F., Offenhauser, N., Dirnagl, U., Priller, J., and Lindauer, U. (2010) Pericytes in capillaries are contractile in vivo, but arterioles mediate functional hyperemia in the mouse brain, Proceedings of the National Academy of Sciences USA 107, 22290-22295.

24. McCaslin, A. F., Chen, B. R., Radosevich, A. J., Cauli, B., and Hillman, E. M. (2010) In vivo 3D morphology of astrocyte-vasculature interactions in the somatosensory cortex: implications for neurovascular coupling, Journal of Cerebral Blood Flow \& Metabolism 31, 795-806.

25. Nishimura, N., Rosidi, N. L., Iadecola, C., and Schaffer, C. B. (2010) Limitations of collateral flow after occlusion of a single cortical penetrating arteriole, Journal of Cerebral Blood Flow \& Metabolism 30, 1914-1927.

26. Stefanovic, B., Hutchinson, E., Yakovleva, V., Schram, V., Russell, J. T., Belluscio, L., Koretsky, A. P., and Silva, A. C. (2007) Functional reactivity of cerebral capillaries, Journal of Cerebral Blood Flow \& Metabolism 28, 961-972.

27. Hutchinson, E. B., Stefanovic, B., Koretsky, A. P., and Silva, A. C. (2006) Spatial flow-volume dissociation of the cerebral microcirculatory response to mild hypercapnia, Neuroimage 32, 520530 .

28. Petzold, G. C., Albeanu, D. F., Sato, T. F., and Murthy, V. N. (2008) Coupling of neural activity to blood flow in olfactory glomeruli is mediated by astrocytic pathways, Neuron 58, 879-910.

29. Lecoq, J. L., Tiret, P., Najac, M., Sheperd, G. M., Greer, C. A., and Charpak, S. (2009) Odorevoked oxygen consumption by action potential and synaptic transmission in the olfactory bulb, Journal of Neuroscience 29, 1424-1433.

30. Chaigneau, E., Oheim, M., Audinat, E., and Charpak, S. (2003) Two-photon imaging of capillary blood flow in olfactory bulb glomeruli, Proceedings of the National Academy of Sciences USA 100, 13081-13086.

31. Chaigneau, E., Tiret, P., Lecoq, J., Ducros, M., Knöpfel, T., and Charpak, S. (2007) The relationship between blood flow and neuronal activity in the rodent olfactory bulb, Journal of Neuroscience 27, 6452-6460.

32. Kobat, D., Durst, M. E., Nishimura, N., Wong, A. W., Schaffer, C. B., and Xu, C. (2009) Deep tissue multiphoton microscopy using longer wavelength excitation, Optics Express 17, 1335413364.

33. Mittmann, W., Wallace, D. J., Czubayko, U., Herb, J. T., Schaefer, A. T., Looger, L. L., Denk, W., and Kerr, J. N. (2011) Two-photon calcium imaging of evoked activity from L5 somatosensory neurons in vivo, Nature Neuroscience 14, 1089-1093. 
34. Kasischke, K. A., Lambert, E. M., Panepento, B., Sun, A., Gelbard, H. A., Burgess, R. W., Foster, T. H., and Nedergaard, M. (2011) Two-photon NADH imaging exposes boundaries of oxygen diffusion in cortical vascular supply regions, Journal of Cerebral Blood Flow \& Metabolism 31, 68-81.

35. Murphy, T. H., Li, P., Betts, K., and Liu, R. (2008) Two-photon imaging of stroke onset in vivo reveals that NMDA-receptor independent ischemic depolarization is the major cause of rapid reversible damage to dendrites and spines, Journal of Neuroscience 28, 756-772.

36. Shih, A. Y., Driscoll, J. D., Drew, P. J., Nishimura, N., Schaffer, C. B., and Kleinfeld, D. (2012) Two-photon microscopy as a tool to study blood flow and neurovascular coupling in the rodent brain, Journal of Cerebral Blood Flow \& Metabolism, 32, 1277-1309.

37. Tsai, P. S., and Kleinfeld, D. (2009) In vivo two-photon laser scanning microscopy with concurrent plasma-mediated ablation: Principles and hardware realization, In Methods for In Vivo Optical Imaging, 2nd edition (Frostig, R. D., Ed.), pp 59-115, CRC Press, Boca Raton.

38. Driscoll, J. D., Shih, A. Y., S. Iyengar, Field, J. J., White, G. A., Squier, J. A., Cauwenberghs, G., and Kleinfeld, D. (2011) Photon counting, censor corrections, and lifetime imaging for improved detection in two-photon microscopy, Journal of Neurophysiology 104, 1803-1811.

39. Nguyen, Q.-T., Dolnick, E. M., Driscoll, J., and Kleinfeld, D. (2009) MPScope 2.0: A computer system for two-photon laser scanning microscopy with concurrent plasma-mediated ablation and electrophysiology, In Methods for In Vivo Optical Imaging, 2nd edition (Frostig, R. D., Ed.), pp 117-142, CRC Press, Boca Raton.

40. Nguyen, Q.-T., Tsai, P. S., and Kleinfeld, D. (2006) MPScope: A versatile software suite for multiphoton microscopy, Journal of Neuroscience Methods 156, 351-359.

41. Drew, P. J., Blinder, P., Cauwenberghs, G., Shih, A. Y., and Kleinfeld, D. (2010) Rapid determination of particle velocity from space-time images using the Radon transform, Journal of Computational Neuroscience 29, 5-11.

42. Valmianski, I., Shih, A. Y., Driscoll, J., Matthews, D. M., Freund, Y., and Kleinfeld, D. (2010) Automatic identification of fluorescently labeled brain cells for rapid functional imaging, Journal of Neurophysiology 104, 1803-1811.

43. Kleinfeld, D., and Mitra, P. P. (2011) Applications of spectral methods in functional brain imaging, In Imaging: A Laboratory Manual (Yuste, R., Ed.), pp 12.11-12.17, Cold Spring Harbor Laboratory Press, New York.

44. Driscoll, J. D., Shih, A. Y., Drew, P. J., Cauwenberghs, G., and Kleinfeld, D. (2011) Two-photon imaging of blood flow in cortex, In Imaging in Neuroscience: A Laboratory Manual (Helmchen, F., Konnerth, A., and Yuste, R., Eds.), pp 927-938, Cold Spring Harbor Laboratory Press, New York.

45. Tsai, P. S., Blinder, P., Kaufhold, J. P., Squier, J. D., and Kleinfeld, D. (2011) All-optical, in situ histology of brain tissue with femtosecond laser pulses, In Imaging in Neuroscience: A Laboratory Manual (Helmchen, F., Konnerth, A., and Yuste, R., Eds.), pp 437-446, Cold Spring Harbor Laboratory Press, New York.

46. Tsai, P. S., Friedman, B., Ifarraguerri, A. I., Thompson, B. D., Lev-Ram, V., Schaffer, C. B., Xiong, Q., Tsien, R. Y., Squier, J. A., and Kleinfeld, D. (2003) All-optical histology using ultrashort laser pulses, Neuron 39, 27-41.

47. Tsai, P. S., Kaufhold, J., Blinder, P., Friedman, B., Drew, P., Karten, H. J., Lyden, P. D., and Kleinfeld, D. (2009) Correlations of neuronal and microvascular densities in murine cortex revealed by direct counting and colocalization of cell nuclei and microvessels, Journal of Neuroscience 18, 14553-14570.

48. Ragan, T., Sylvan, J. D., Kim, K. H., Huang, H., Bahlmann, K., Lee, R. T., and So, P. T. (2007) High-resolution whole organ imaging using two-photon tissue cytometry, Journal of Biomedical Optics 12, 014015. 
49. Kleinfeld, D., and Delaney, K. R. (1996) Distributed representation of vibrissa movement in the upper layers of somatosensory cortex revealed with voltage sensitive dyes, Journal of Comparative Neurology 375, 89-108.

50. Levasseur, J. E., Wei, E. P., Raper, A. J., Kontos, A. A., and Patterson, J. L. (1975) Detailed description of a cranial window technique for acute and chronic experiments, Stroke 6, 308-317.

51. Morii, S., Ngai, A. C., and Winn, H. R. (1986) Reactivity of rat pial arterioles and venules to adenosine and carbon dioxide: With detailed description of the closed cranial window technique in rats, Journal of Cerebral Blood Flow \& Metabolism 6, 34-41.

52. Tuor, U. I., Simone, C. S., Barks, J. D., and Post, M. (1993) Dexamethasone prevents cerebral infarction without affecting cerebral blood flow in neonatal rats, Stroke 24, 452-457.

53. Dombeck, D. A., Graziano, M. S., and Tank, D. W. (2009) Functional clustering of neurons in motor cortex determined by cellular resolution imaging in awake behaving mice, Journal of Neuroscience 29, 13751-13760.

54. Holtmaat, A., Bonhoeffer, T., Chow, D. K., Chuckowree, J., De Paola, V., Hofer, S. B., Hübener, M., Keck, T., Knott, G., Lee, W. C., Mostany, R., Mrsic-Flogel, T. D., Nedivi, E., PorteraCailliau, C., Svoboda, K., Trachtenberg, J. T., and Wilbrecht, L. (2009) Long-term, highresolution imaging in the mouse neocortex through a chronic cranial window, Nature Protocols 4 , 1128-1144.

55. Mostany, R., and Portera-Cailliau, C. (2008) A method for 2-photon imaging of blood flow in the neocortex through a cranial window, Journal of Visualized Experiments 12, 678.

56. Yang, G., Pan, F., Parkhurst, C. N., Grutzendler, J., and Gan, W. B. (2010) Thinned-skull cranial window technique for long-term imaging of the cortex in live mice, Nature Protocols 5, 201-208.

57. Drew, P. J., Shih, A. Y., Driscoll, J. D., Knutsen, P. M., Davalos, D., Blinder, P., Akassoglou, K., Tsai, P. S., and Kleinfeld, D. (2010) Chronic optical access through a polished and reinforced thinned skull, Nature Methods 7, 981-984.

58. Shih, A. Y., Drew, P. J., Mateo, C., Tsai, P. S., and Kleinfeld, D. (2012) A polished and reinforced thinned skull window for long-term imaging and optical manipulation of the mouse cortex, Journal of Visualized Experiments, http://www.jove.com/video/3742.

59. Frostig, R. D., Lieke, E. E., Ts'o, D. Y., and Grinvald, A. (1990) Cortical functional architecture and local coupling between neuronal activity and the microcirculation revealed by in vivo highresolution optical imaging of intrinsic signals, Proceedings of the National Academy of Sciences USA 87, 6082-6086.

60. Grinvald, A., Lieke, E. E., Frostig, R. D., Gilbert, C. D., and Wiesel, T. N. (1986) Functional architecture of cortex revealed by optical imaging of intrinsic signals, Nature 324, 361-364.

61. Ayling, O. G., Harrison, T. C., Boyd, J. D., Goroshkov, A., and Murphy, T. H. (2009) Automated light-based mapping of motor cortex by photoactivation of channelrhodopsin-2 transgenic mice, Nature Methods 6, 219-224.

62. Mayhew, J. E. W., Askew, S., Zeng, Y., Porrill, J., Westby, G. W. M., Redgrave, P., Rector, D. M., and Harper, R. M. (1996) Cerebral vasomotion: $0.1 \mathrm{~Hz}$ oscillation in reflectance imaging of neural activity., Neuroimage 4, 183-193.

63. Rovainen, C. M., Woolsey, T. A., Blocher, N. C., Wang, D.-B., and Robinson, O. F. (1993) Blood flow in single surface arterioles and venules on the mouse somatosensory cortex measured with videomicroscopy, fluorescent dextrans, nonoccluding fluorescent beads, and computer-assisted image analysis, Journal of Cerebral Blood Flow \& Metabolism 13, 359-371.

64. Stosiek, C., Garaschuk, O., Holthoff, K., and Konnerth, A. (2003) In vivo two-photon calcium imaging of neuronal networks, Proceedings of the National Academy of Sciences USA 100, 73197324.

65. Garaschuk, O., Milos, R. I., and Konnerth, A. (2006) Targeted bulk-loading of fluorescent indicators for two-photon brain imaging in vivo, Nature Protocols 1, 380-386.

66. Nimmerjahn, A., Kirchhoff, F., Kerr, J. N., and Helmchen, F. (2004) Sulforhodamine 101 as a specific marker of astroglia in the neocortex in vivo, Nature Methods 29, 31-37. 
67. Kim, T., and Kim, S. G. (2011) Temporal dynamics and spatial specificity of aterial and venous blood volume changes during visual stimulation: Implication for BOLD quantification, Journal of Cerebral Blood Flow \& Metabolism 31, 1211-1222.

68. Buxton, R. B., Wong, E. C., and Frank, L. R. (1998) Dynamics of blood flow and oxygenation changes during brain activation: The balloon model., Magnetic Resonance in Medicine 39, 855864.

69. Drew, P. J., Duyn, J. H., Galanov, E., and Kleinfeld, D. (2008) Finding coherence in spontaneous oscillations, Nature Neuroscience 11, 991-993.

70. Helmchen, F., and Denk, W. (2005) Deep tissue two-photon microscopy, Nature Methods 2, $932-$ 940.

71. Botcherby, E. J., Smith, C. W., Kohl, M. M., Débarre, D., Booth, M. J., Juškaitis, R., Paulsen, O., and Wilson, T. (2012) Aberration-free three-dimensional multiphoton imaging of neuronal activity at kHz rates, Proceedings of National Academy of Sciences USA 109, 2919-2924.

72. Sohya, K., Kameyama, K., Yanagawa, Y., Obata, K., and Tsumoto, T. (2007) GABAergic neurons are less selective to stimulus orientation than excitatory neurons in layer II/III of visual cortex, as revealed by in vivo functional $\mathrm{Ca}^{2+}$ imaging in transgenic mice, Journal of Neuroscience 27, 2145-2149.

73. Tian, L., Hires, S. A., Mao, T., Huber, D., Chiappe, M. E., Chalasani, S. H., Petreanu, L., Akerboom, J., McKinney, S. A., Schreiter, E. R., Bargmann, C. I., Jayaraman, V., Svoboda, K., and Looger, L. L. (2009) Imaging neural activity in worms, flies and mice with improved GCaMP calcium indicators, Nature Methods 6, 875-881.

74. Lee, J. H., Durand, R., Gradinaru, V., Zhang, F., Goshen, I., Kim, D. S., Fenno, L. E., Ramakrishnan, C., and Deisseroth, K. (2010) Global and local fMRI signals driven by neurons defined optogenetically by type and wiring, Nature 465, 788-792.

75. Alexander, G. M., Rogan, S. C., Abbas, A. I., Armbruster, B. N., Pei, Y., Allen, J. A., Nonneman, R. J., Hartmann, J., Moy, S. S., Nicolelis, M. A., McNamara, J. O., and Roth, B. L. (2009) Remote control of neuronal activity in transgenic mice expressing evolved $\mathrm{G}$ protein-coupled receptors, Neuron 63, 27-39.

76. Freund, Y. (2009) A more robust boosting algorithm, arXive, Arxiv/0905.2138

77. Di Caro, G., and Dorigo, M. (1998) AntNet: Distributed stigmergetic control for communications networks, Journal of Artificial Intelligence Research 9, 317--365. 


\section{Figure Legends}

Figure 1. Intrinsic optical signal imaging for functional region targeting. A female Long Evans adult ( 2 month) rat was anesthetized with isoflurane $\left(2 \%\right.$ in $\mathrm{O}_{2}$ for induction and $<1 \%$ sustained) and a $4 \mathrm{~mm}$ x $4 \mathrm{~mm}$ closed craniotomy performed over the vibrissa area of primary somatosensory cortex. The IOS was obtained as the reflectance at $633 \mathrm{~nm}$ as a function of time and trial. The sampling period is $50 \mathrm{~ms}$, the pixel width is $8 \mu \mathrm{m}$, the duty cycle for each trial was $18 \mathrm{~s}$, and we obtained 30 trials. (A) An example of the IOS for deflection of the $\mathrm{C} 2$ vibrissa, realized as $\mathrm{a} \pm 12^{\circ}$ movement by $10 \mathrm{~Hz}$ square wave filtered with a $6^{\text {th }}$ order $100 \mathrm{~Hz}$ Bessel low pass filter. The dark declivity indicates reduced reflectance of red light, suggesting an increase of deoxygenated hemoglobin and thus increased neural activity in that region. (B) Intrinsic signal for a profile line through the centroid of the activated region in panel A for three separate blocks of trials. The horizontal dashed lines are a $50 \%$ decrease of the intrinsic signal; the solid vertical lines indicate the centroids. (C) The thresholded IOS image, e.g., dashed line in panel $\mathrm{B}$, for 14 different vibrissae across the cortical surface. The surface map was obtained by reflectance at $475 \mathrm{~nm}$. Thresholds values were $\mathrm{B} 1=-1, \mathrm{~B} 2=-2.5, \mathrm{~B} 3=0, \mathrm{C} 1=-2, \mathrm{C} 2=-1, \mathrm{C} 3=0, \mathrm{C} 4=-1.5, \mathrm{D} 1=-2, \mathrm{D} 2=-1, \mathrm{D} 3=-1.5, \mathrm{D} 4=-3, \mathrm{E} 1=-$ $0.5, \mathrm{E} 2=-3, \mathrm{E} 3=0 ;$ all $\mathrm{x} 10^{-4}$. In some areas the vessels were masked from the calculation, e.g., vibrissa D2.

Figure 2. Simultaneous measurement of diameter and velocity in two vessels using spatially optimized line scans. (A) Image of fluorescently stained vessels in somatosensory cortex of a Sprague Dawley rat. The forelimb and hindlimb representations across cortex were mapped using intrinsic optical imaging, similar to that in figure 1. (B) Image of a surface arteriole and venule, with scan pattern superimposed. Portions of the scan path along the length are used to calculate RBC velocity, while portions across the diameter of the vessels are used to calculate diameter. Scans were acquired at a rate of 735 lines/s. (C) Scan path, colored to show the error between the desired scan path and the actual path the mirrors traversed. The error along linear portions of the image is about $1 \mu \mathrm{m}$, and increases when the 
mirrors undergo rapid acceleration. The error between successive scans of the same path is less than $0.15 \mu \mathrm{m}$, several times lower than the point spread function for two-photon microscopy. (D) Scan mirror speed as a function of time (top). Note that portions used to acquire diameter and velocity data are constant speed (top). The line scans generated from the path can be stacked sequentially as a function of time to produce a raw cascade image (bottom). (E) Vessel diameter is calculated as the full width at half maximum of a time average of several scans across the width of a vessel (left). Red blood cell velocity calculated from the angle of the streaks caused by the flow of RBCs. (F) Data traces of diameter, velocity, and flux for the arteriole and venule, processed to remove heart rate and smoothed with a running window. Both vessels show an increase in flux in response to forelimb stimulation. In the arteriole, this flux increase is due to simultaneous increase of lumen diameter and RBC velocity. In contrast, flux increase in the venule is due only to an increase in RBC velocity, as diameter is unchanged by stimulation. All panels adapted from Driscoll et al. (44).

Figure 3. Example of automated cell segmentation and user-defined fast scanning for functional imaging in rat parietal cortex. (A) A full field image of 19 neurons (N), 1 astrocyte (A), and 3 blood vessels, obtained at 4 frames/s, with a scan path superimposed on cells determined by our machine learning algorithm. All cells and vessels are scanned at $110 \mathrm{~Hz}$. The green channel shows the fluorescence from OGB-1 and fluorescein while the red channel shows fluorescence from SR101. (B) Activity of neurons and an astrocyte, indicated in panel $\mathrm{A}$, in response to a single weak electrical shock to the

forelimb. (C) The $\mathrm{Ca}^{2+}$ response of the astrocyte (A1), the average neuronal response (N1 - N19), and the speed of red blood cells in one capillary (V1). All panels adapted from Valmianski et al. (42).

Figure 4. Spontaneous and stimulus-induced vascular dynamics in the cortex of awake mouse. (A) Schematic of the experimental setup. The awake mouse is head fixed by means of a bolt and sits passively in an acrylic cylinder beneath the two-photon microscope. Air puffers for sensory stimulation are aimed at 
the vibrissa and as a control at the tail. (B) Individual dilation responses to $30 \mathrm{~s}$ vibrissae stimulation. (C) Plot of peak averaged dilation responses to $30 \mathrm{~s}$ vibrissae stimulation. Early arterial peaks, in the 0 to $10 \mathrm{~s}$ interval after stimulation, are denoted by red circles; regression slope $=0.007 \mu \mathrm{m}^{-1}\left(\mathrm{r}^{2}=0.15, \mathrm{p}<0.02\right)$. Late arterial peaks, greater than $10 \mathrm{~s}$ after onset, are denoted by red triangles; the linear regression (not shown) is not significant. Venules are denoted by blue dots; the linear regression is not significant. (D) Plot of peak value of the spontaneous dilations for arteries, in red, and veins, in blue. Grey area shows the $0.2 \mu \mathrm{m}$ resolution limit of detectable changes. Lines show linear regressions; slope $=-0.004 \mu \mathrm{m}^{-1}$ for arterioles is significant $\left(\mathrm{r}^{2}=0.13, \mathrm{p}<0.001\right)$, while that for veins (not shown) is not significantly different from zero. All panels adapted from Drew et al. (16).

Figure 5. Deep imaging of cortical angioarchitecture and blood flow. (A) Maximum intensity projections of mouse cortical vasculature in the coronal orientation. The image stack was collected over the entire depth of cortex through a cranial window with the dura intact. To reduce scattering and improve imaging depth, a long wavelength of excitation, i.e., $1280 \mathrm{~nm}$, was used and the blood plasma was labeled by intravenous injection of Alexa 680 conjugated to dextran.. (B) Single planar image taken from panel A at the depth of the red line. (C) Magnified image taken from a vessel from the region in the red box in panel B. (D) Magnified image taken from a vessel from the region in the red box in panel B. All panels adapted from Kobat et al. (32).

Figure 6. Examples of cell segmentation and fast scanning for functional imaging of neurons, astrocytes, and vessels in rat parietal cortex. (A) A full-field image of 21 neurons, 1 astrocyte, and 3 blood vessels, obtained at 4 frames/s, with a scan path superimposed on it in which all cells are sampled at $110 \mathrm{~Hz}$. The green channel shows the fluorescence from Oregon Green Bapta-1 while the red channel shows fluorescence from Sulforhodamine 101. White shows the outlines of cells as determined by our 
automated algorithm. The scan path is superimposed on the image; solid lines are sections of the scan used to record responses while dashed lines are section of high scan mirror acceleration. (B) Activity of selected neurons and the astrocyte indicated in panels $\mathrm{E}$ and $\mathrm{F}$ during the same time interval as shown in panel F. (C) The average neuronal calcium response across 19 of the 2 neurons (N1 - N19), the calcium response of the astrocyte (A1),and the speed of red blood cells in one capillary (V1). All panels adapted from Valmianski et al. (42). 

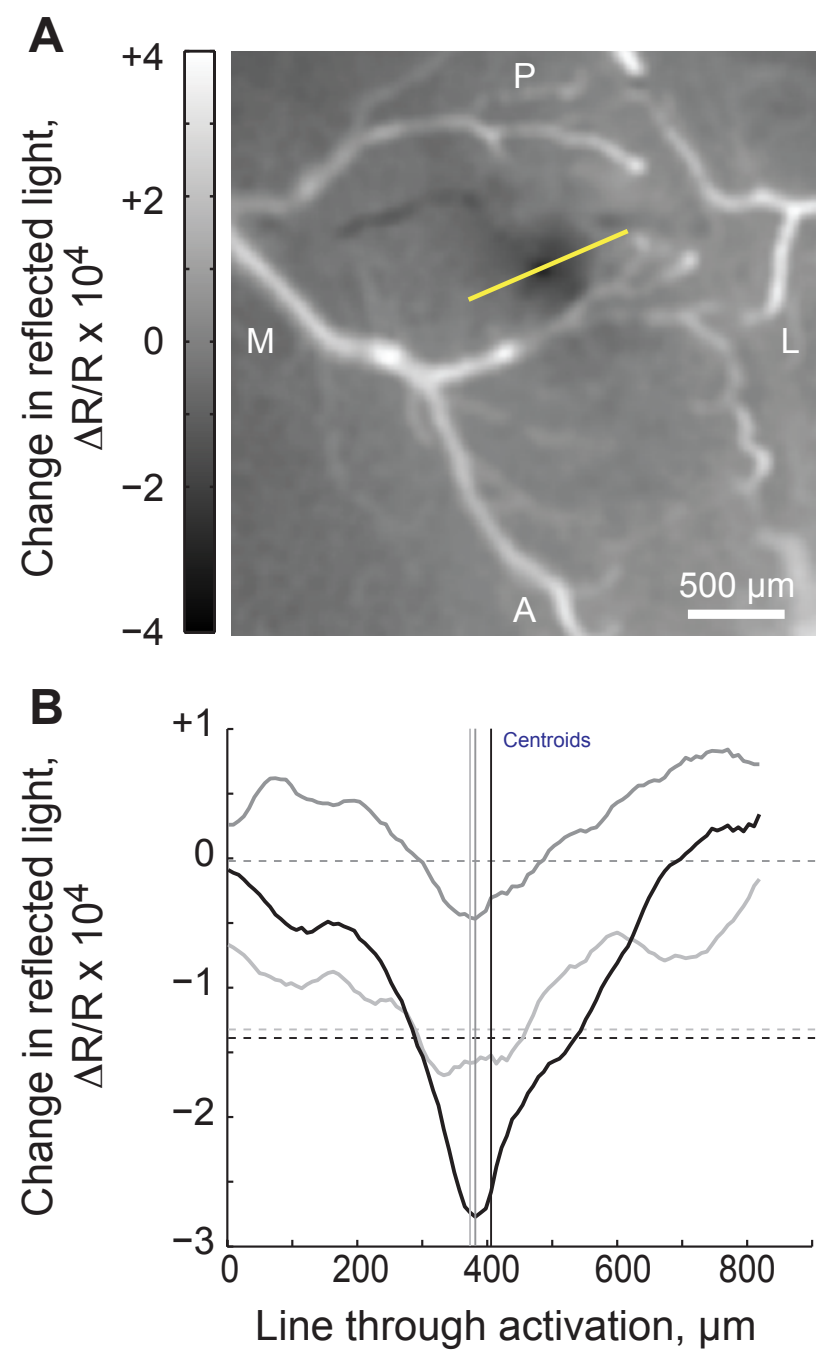

C

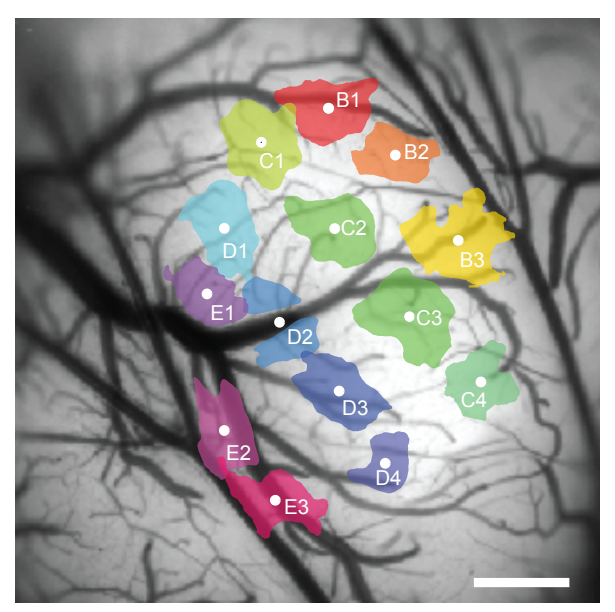

Figure 1 - Shih, Driscoll, Pesavento and Kleinfeld (2012) 

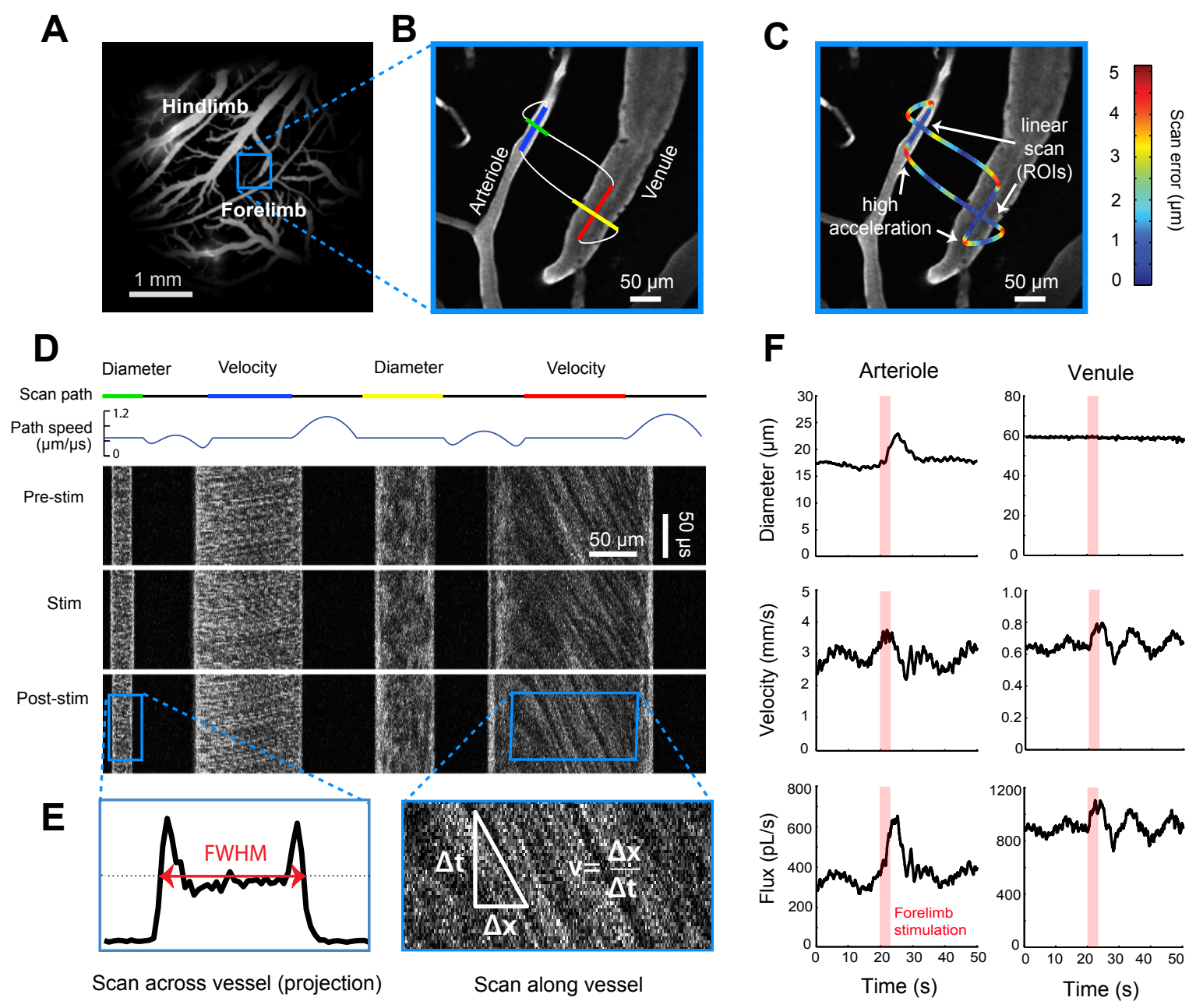

Figure 2 - Shih, Driscoll, Pesavento and Kleinfeld (2012) 
A

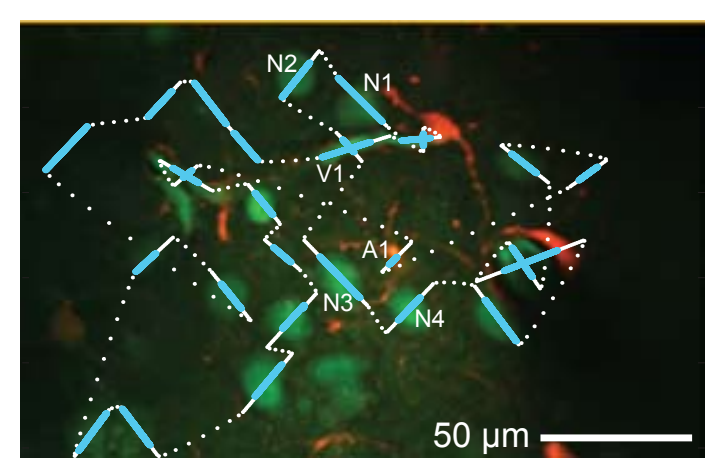

\section{B}

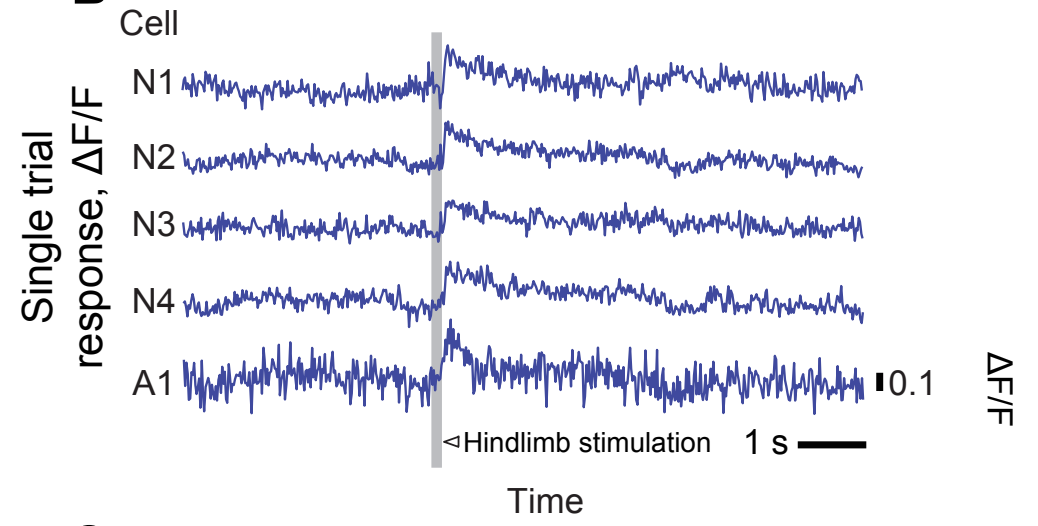

C All neurons

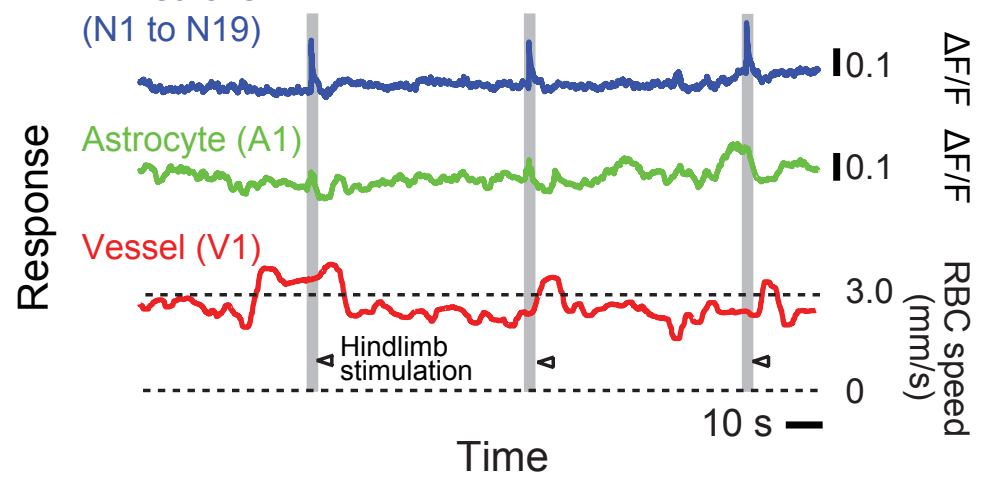

Figure 3 - Shih, Driscoll, Pesavento and Kleinfeld (2012) 

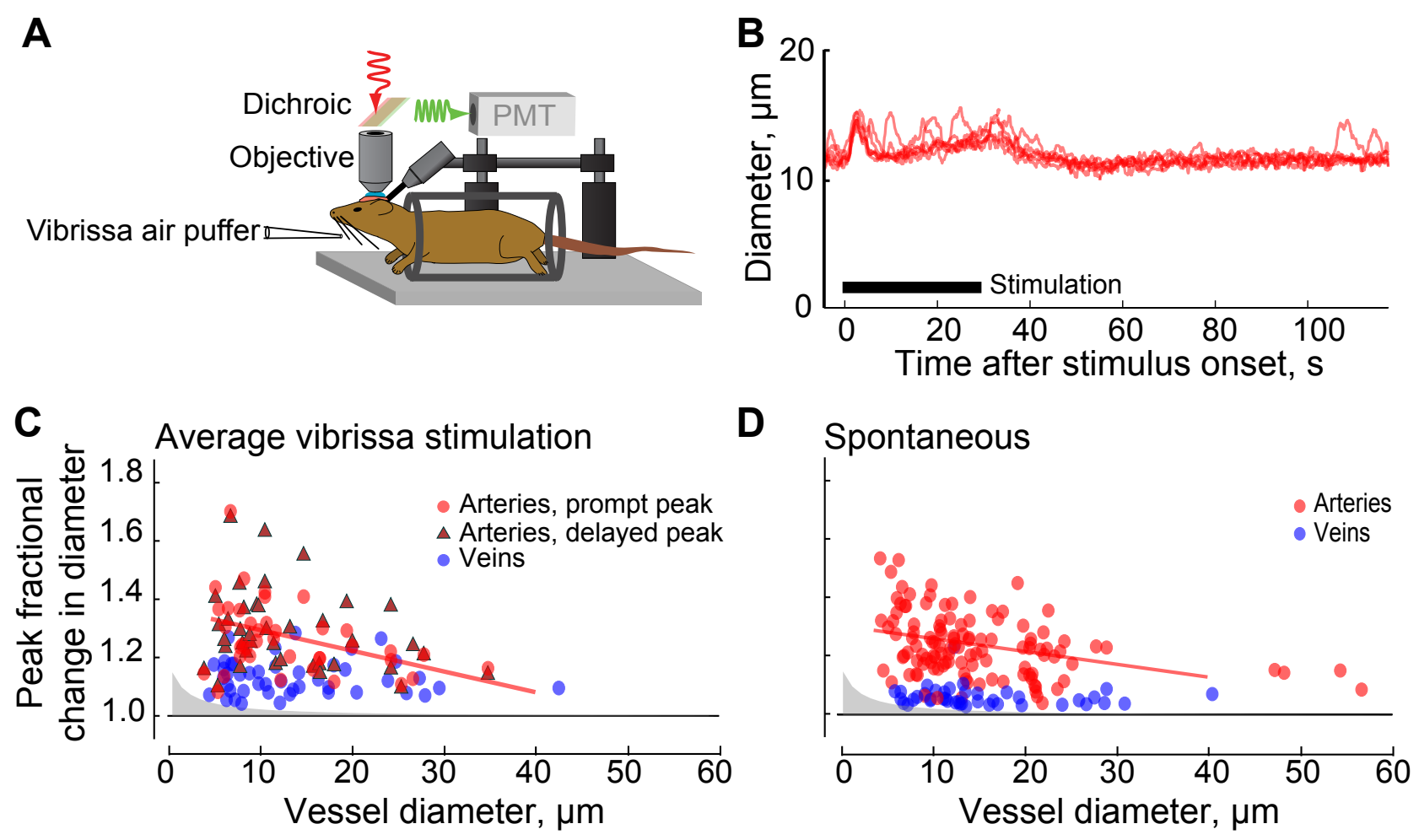


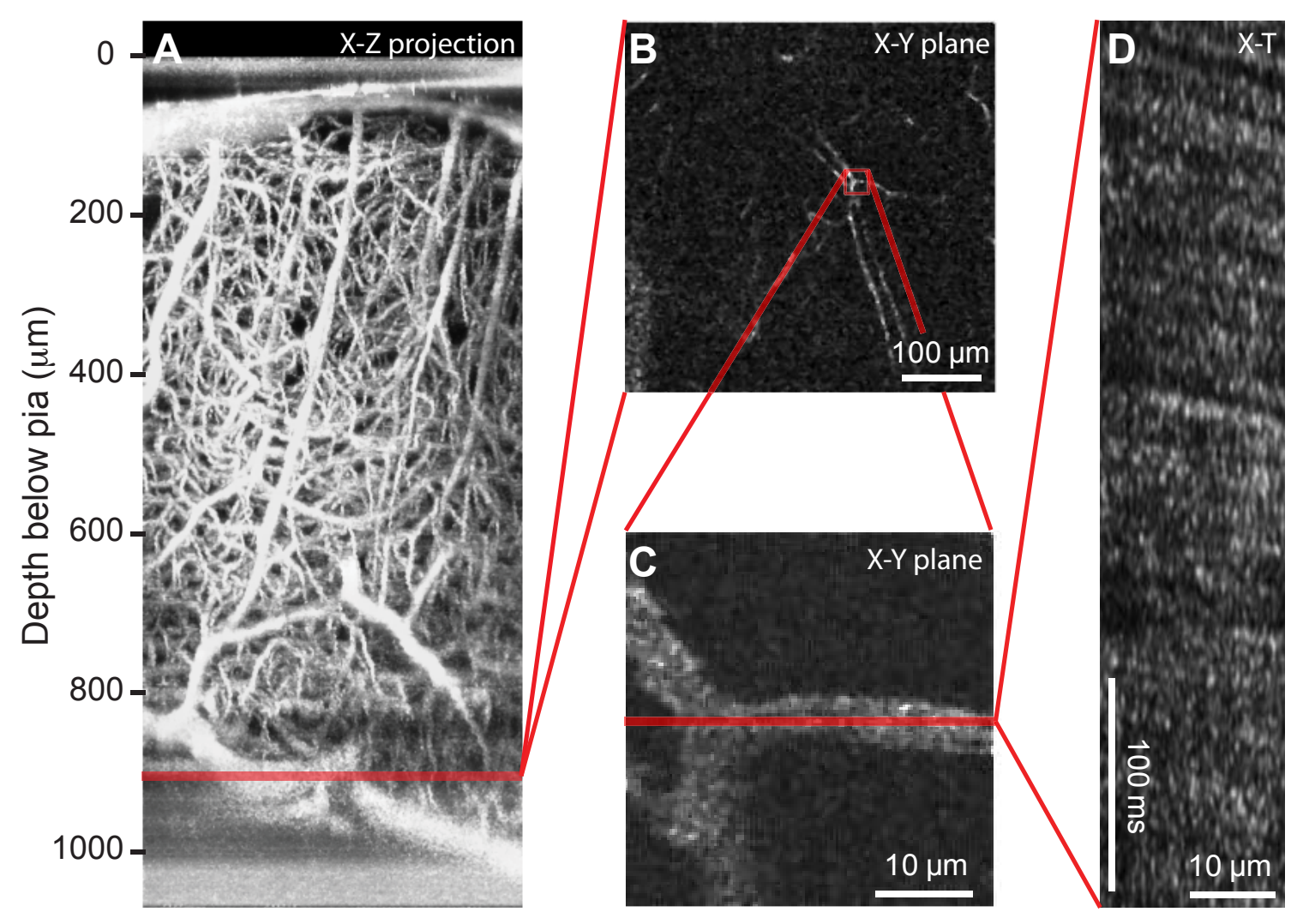

Figure 5 - Shih, Driscoll, Pesavento and Kleinfeld (2012) 